\title{
Big Extra Dimensions Make $\Lambda$ too Small
}

\author{
Rafael D. Sorkin \\ Perimeter Institute, 31 Caroline Street North, \\ Waterloo ON, N2L 2 Y5 Canada \\ and \\ Department of Physics, Syracuse University, \\ Syracuse, NY 13244-1130, U.S.A. \\ Received on 23 February, 2005
}

\begin{abstract}
I argue that the true quantum gravity scale cannot be much larger than the Planck length, because if it were then the quantum gravity-induced fluctuations in $\Lambda$ would be insufficient to produce the observed cosmic "dark energy". If one accepts this argument, it rules out scenarios of the "large extra dimensions" type. I also point out that the relation between the lower and higher dimensional gravitational constants in a Kaluza-Klein theory is precisely what is needed in order that a black hole's entropy admit a consistent higher dimensional interpretation in terms of an underlying spatio-temporal discreteness.
\end{abstract}

Probably few people anticipate that laboratory experiments which can be done in the foreseeable future will help to guide the construction of a theory of quantum gravity. Even astronomers, who can access a much greater range of conditions than we can reproduce here on earth, have hardly provided relevant observations so far, and only in one or two cases has any of their data been compared with the predictions of any quantum gravity theory. The reason for expecting this experimental impotence to persist, of course, is that quantum gravitational effects are normally taken to be associated with a length scale of around $10^{-32} \mathrm{~cm}$, whereas our laboratory instruments are only able to probe distances which are bigger than that, by fifteen or more orders of magnitude.

But how sure are we that the quantum gravity scale is genuinely set by the gravitational coupling constant $\kappa=8 \pi G$ (together with $\hbar$ and $c$, taken as unity)? Although this is by far the most natural assumption, no one knows with certainty that it is correct. On the contrary, a variety of authors have proposed in recent years that the "true Planck length" is much bigger than we think it is, possibly big enough to be accessible to the next generation of particle colliders. In these alternative scenarios, the $G$ that we derive from planetary orbits and the like is not supposed to be fundamental, because our spacetime is supposed to be only a submanifold ("brane") embedded in some higher dimensional spacetime, and it is the Planck length associated with this higher dimension's gravity that would be truly fundamental.

Were one of these modified Kaluza-Klein scenarios correct, quantum gravity might be about to enter an experimental paradise like the one in which particle physics thrived a few decades ago. However, there is an irony in the situation, as I will try to show in this paper [1].

An enlargement of the Planck length sufficient to bring all these benefits would end up depriving us of the one predictive success that quantum gravity has had so far, namely that concerning the cosmological constant $\Lambda$. More specifically, what I will argue is that: if the fluctuations [3] predicted by causal set theory give a true account of the non-zero $\Lambda$ ("dark energy"), and if the fundamental discreteness hypothesized by causal sets corresponds to the scale set by some more fundamental, higher dimensional gravitational constant, then making the latter much bigger than $G$ would predict fluctuations in $\Lambda$ far too small to be compatible with its observed value. That is, the "large extra dimension" scenarios would be ruled out on this basis.

Underlying this conclusion is the intuition that the fundamental length posited by causal set theory, or any other discrete theory, must have an order of magnitude given by the (true) gravitational coupling constant $\kappa$. Or to put it the other way around, one is supposing that the dimensional constant $\kappa$ is more or less directly reflecting not, for example, some length-parameter of the standard model of particle physics, but rather a more fundamental length or "cutoff" in nature at which the continuum picture breaks down. In much the same manner - kinetic theory tells us - the molecular lengthand time-scales set the order of magnitude of such continuum "coupling constants" as the diffusion constant and the speed of sound. Indeed, one can take the point of view that without some discrete structure underlying spacetime, there would be no good reason for the concept of length to exist at all.

This kind of intuition or "dimensional analysis" is bolstered by one's experience with renormalization in quantum field theory and in statistical mechanics, more specifically by experience built up in connection with the so-called "renormalization group".

But perhaps its strongest support in the gravitational context comes from our understanding of black hole thermodynamics, which almost forces on us the idea [4] that the entropy will ultimately be understood in terms of some underlying discrete structures "occupying" roughly one unit of horizon area each, where, in order to match the formula $S=2 \pi A / \kappa$, the unit of area must be that corresponding to a length of $l \sim \sqrt{\kappa}$.

The above parameters $\kappa$ and $l$ refer specifically to four dimensions, but they generalize immediately to dimension $D=4+d$. There, the (rationalized) gravitational coupling constant $\kappa$ has dimensions of $[\text { length }]^{D-2}$ (in order that the term in the gravitational Action $\frac{1}{2 \kappa} \int R d V$ be dimensionless) and so we may assume that

$$
\kappa \sim l^{D-2} \sim l^{d+2},
$$

where $l$ is the fundamental length of the higher dimensional theory.

Having identified the fundamental length-scale, we must now relate it to the cosmological constant. Here we merely 
reproduce the heuristic conclusion from causal set theory that, at any given cosmological epoch, and in Planckian units,

$$
\Lambda \sim N^{-1 / 2}
$$

where $N$ is the number [5] of elements since the "big bang" $[3,6]$. This long-standing prediction correctly yielded the observed order of magnitude of the so-called "dark energy" and it also explains why $\Lambda$ should coincide in order of magnitude with the density of ambient matter. For purposes of the present analysis, I will assume that (2) is valid in general, even though a full comparison of this idea with the cosmological data has not yet been completed. In this relationship, the number $N$ is to be identified, according to one of the basic principles of causal set theory, with the spacetime volume in fundamental units (up to an unknown multiplicative constant of order unity), that is:

$$
N \sim V / l^{n},
$$

where $n$ is the spacetime dimensionality and

$$
V=\int d V=\int d^{n} x|g|^{\frac{1}{2}} .
$$

From these equations, it is clear that changing the fundamental length $l$ will change the predicted value of $\Lambda$. We will see that in the scenarios under consideration, this change will lead to a current value that is much too small.

The models in question are, more specifically, those in which the quantum gravity energy scale is brought down to something on the order of a $\mathrm{TeV}$, or in any case much lower than the normal Planck scale. Given the bemusing variety of such models, it seems difficult to make any blanket statement covering all of them. I will thus limit myself to three which seem to be the sources of most of the others, namely [7-9]. In fact, I will limit myself primarily to the the first of these, because the analysis is more straightforward for it. I believe that the conclusions would be the same for the other two models, but I am not certain, because the physical interpretations of these models are less clear to me, especially in the case of [8].

In the following, we will be comparing predictions from the usual four dimensional theory with predictions from a hypothetical, higher dimensional model. To distinguish between analogous quantities in the two cases, I will use a tilde. Thus, for example $\kappa=\sqrt{8 \pi G}$ will be the usual gravitational constant, while $\tilde{\kappa}$ will be the analogous higher dimensional parameter, i.e. the one which is more fundamental if the theory is true. Similarly $l$ and $\tilde{l}$ will be the corresponding fundamental length-scales.

Recall that in the original Kaluza-Klein models, spacetime was taken to be, at least locally [10], a product manifold of the form $\tilde{M}=M \times K$, where $K$ is some compact "internal space", in the first instance a circle. The effective spacetime $M$ after coarse-graining to distance scales much longer than the compactification diameter must thus be identified with the quotient of the higher dimensional spacetime with respect to $K$. (From time to time, people would write papers in which they identified our spacetime with a submanifold rather than a quotient, but that was only because they didn't understand the theory!)
In these models, one sees clearly how the four and five (or higher) dimensional gravitational constants are related. Suppose for example that the internal space $K$ is a circle or some other Ricci-flat manifold like a torus. Then the gravitational Action written in terms of the higher dimensional metric on $\tilde{M}$ will be

$$
S_{\text {grav }}=\frac{1}{2 \tilde{\kappa}} \int \tilde{R} d \tilde{V}
$$

while in terms of the dimensionally reduced metric on $M$ it will be

$$
S_{\text {grav }}=\frac{1}{2 \kappa} \int R d V
$$

Since in this case we have $R=\tilde{R}$, equating the last two expressions yields $d V / \kappa=d \tilde{V} / \tilde{\kappa}$ or equivalently

$$
\tilde{\kappa}=v \kappa
$$

because $d \tilde{V}=v d V$, where ' $v$ ' represents the volume of the internal manifold $K$. (Really we should be writing approximate rather than exact inequalities here, because the renormalization of $\kappa$ and $\tilde{\kappa}$ has been ignored. Indeed all quantum effects have been ignored in assuming that the product metric is governed by the classical Einstein equations.) Converting this relationship between $\kappa$ and $\tilde{\kappa}$ into a relationship between $l$ and $\tilde{l}$ produces, in light of (1),

$$
(\tilde{l} / l)^{2} \sim v / \tilde{l}^{d}
$$

In principle, the true discreteness scale $\tilde{l}$ could thus be very different from what we call the Planck length, if the compactification diameter were very large in fundamental units. However, in the traditional setting, in which $M$ is a quotient of $\tilde{M}$, this would produce gauge coupling constants which were much too small.

In the more recent scenarios, though, $M$ is identified with a 4-dimensional membrane within $\tilde{M}$ (a so-called 3-brane), and the gauge fields are supposed to be confined to this membrane, protecting them from being diluted. It is thus that the possibility arises of " $\mathrm{TeV}$ quantum gravity".

In order to see most simply how this possibility runs into trouble with the cosmological constant, notice that to equate $\Lambda$ to $1 / \sqrt{N}$ in natural units, is equivalently to assert that (in such units)

$$
S_{\Lambda}:=\Lambda V=\Lambda N \sim \sqrt{N}
$$

This puts the content of (2) in truly dimensionless form, since both $S_{\Lambda}$ and $N$ are pure numbers. Now if we change our idea of the fundamental length, we will have to change $N$ as well; and (6) teaches us that the effective cosmological constant $\Lambda$ will then change in the same ratio, or rather its square root [12].

What is this ratio according to the scenario of reference [7]? With the help of equations (3) and (5), we can answer this 
question easily:

$$
\begin{aligned}
\frac{\tilde{N}}{N} & \sim \frac{\tilde{V} / \tilde{l}^{4+d}}{V / l^{4}}=\frac{\tilde{V}}{V} \frac{l^{4}}{\tilde{l}^{4+d}}=\frac{v}{\tilde{l}^{d}}\left(\begin{array}{l}
l \\
\tilde{\tilde{l}}
\end{array}\right)^{4} \\
& \sim\left(\begin{array}{l}
\tilde{l} \\
\bar{l}
\end{array}\right)^{2}\left(\begin{array}{l}
l \\
\overline{\tilde{l}}
\end{array}\right)^{4}=\left(\begin{array}{l}
l \\
\overline{\tilde{l}}
\end{array}\right)^{2}
\end{aligned}
$$

whence

$$
\sqrt{\tilde{N} / N} \sim l / \tilde{l}
$$

With $\tilde{l}$ corresponding to anything like a $T e V$, this yields a $\Lambda$ many orders of magnitude too small to do justice to the supernova data [13].

In concluding, I'd like to return briefly to the basic assumption (1) in relation to the idea that (the magnitude of) a black hole's entropy reflects an underlying discreteness of spacetime. Earlier, I adduced this idea to support the identification of the discreteness scale with the scale set by the gravitational coupling constant. But if this presumed relationship is consistent in four dimensions, it is not obvious a priori that it will also be consistent with respect to the higher dimensional spacetime. If it were not, then one might have to call into question, not only the "large extra dimensions" scenarios, but the Kaluza-Klein paradigm in general. Fortunately, it turns out that Kaluza-Klein theories have no quarrel with spacetime discreteness in this sense.

Consider a black hole (or cosmological) horizon described with respect to the effective, 4-dimensional spacetime $M$. If its area is $A$, then its entropy will be roughly $A / l^{2}, l$ being the fundamental length deduced from the 4-dimensional theory. With respect to the higher dimensional metric, however, the same horizon, being extended over the internal manifold $K$, has a $2+d$-dimensional "area" of $\tilde{A}=A v$. Its entropy should therefore be about $\tilde{A} / \tilde{l}^{2+d}=A v / \tilde{l}^{2+d}$, if $\tilde{l}$ truly sets the higher dimensional discreteness scale. Do these two formulas agree? Clearly, they do if and only if $(\tilde{l} / l)^{2} \sim v / \tilde{l}^{d}$; but this precisely the content of equation (5). Conversely, equation (5) can be understood as the compatibility condition between the lower and higher dimensional ways of "counting horizon molecules". I think that this concordance strengthens the evidence for (1), and to that extent strengthens the evidence for the conclusions we have drawn from it. [If nothing else, it furnishes a useful mnemonic for remembering equation (5)!]

In the previous paragraph, the viewpoint adopted was that of traditional Kaluza-Klein models, for which the lower dimensional horizon is a quotient of the higher dimensional one. This cannot be quite correct in scenarios such as those of [8] and [9], however. It would be interesting to examine the above question of "horizon counting" in those cases too.

\section{Acknowledgments}

For enlightening conversations on the subject matter of this paper, I would like to thank Yujun Chen, Jaume Gomis, Richard Corrado, and Rob Myers.

This research was partly supported by NSF grant PHY0404646 and by funds from the Office of Research and Computing of Syracuse University.
[1] In the conference, my talk was a review of causal set theory overall that would be difficult to reproduce here in the space allotted. (For other overviews, see Refs. [2].) Instead, I have selected one topic from my talk (the cosmological constant) and developed one possible consequence of the ideas presented there.

[2] L. Bombelli, J. Lee, D. Meyer and R.D. Sorkin, "Spacetime as a causal set", Phys. Rev. Lett. 59, 521-524 (1987);

R.D. Sorkin, "Causal Sets: Discrete Gravity (Notes for the Valdivia Summer School)", in the proceedings of the Valdivia Summer School, held January 2002 in Valdivia, Chile, A. Gomberoff and D. Marolf (eds.), (to appear), gr-qc/0309009; D.D. Reid, "Discrete Quantum Gravity and Causal Sets", Canadian Journal of Physics 79, 1-16 (2001), gr-qc/9909075;

R.D. Sorkin, "The causal set as the deep structure of spacetime", Living Reviews in Relativity, AEI, to appear.

[3] R.D. Sorkin, "First Steps with Causal Sets", in: General Relativity and Gravitational Physics, R. Cianci, R. de Ritis, M. Francaviglia, G. Marmo, C. Rubano and P. Scudellaro (eds.), Proceedings of the Ninth Italian Conference of the same name, held Capri, Italy, September, 1990 (World Scientific, Singapore, 1991), pp. 68-90.

R.D. Sorkin, "Forks in the Road, on the Way to Quantum Gravity", talk given at the conference entitled "Directions in General Relativity", held at College Park, Maryland, May, 1993, published in: Int. J. Th. Phys. 36, 2759-2781 (1997), grqc/9706002.

[4] See: R.D. Sorkin, "The Statistical Mechanics of Black Hole Thermodynamics", in: Black Holes and Relativistic Stars, R.M. Wald (ed.) (U. of Chicago Press, 1998), pp. 177-194, grqc/9705006.

[5] The earlier treatments of [3] did not define $N$ precisely. The more complete model of [6] interprets $N$ "at" any given element $x$ as the number of elements causally preceding $x$ and belonging to the current cycle of cosmic expansion. By equation (3) this is given by the volume contained within the past light cone of $x$.

[6] M. Ahmed, S. Dodelson, P. Greene and R.D. Sorkin, "Everpresent $\Lambda$ ”, Phys. Rev. D 69, 103523 (2004), astro-ph/0209274.

[7] N. Arkani-Hamed, S. Dimopoulos and G. Dvali, "The Hierarchy Problem and New Dimensions at a Millimeter", Phys. Lett. B429, 263-272 (1998), hep-ph/9803315.

[8] L. Randall and R. Sundrum, "A Large Mass Hierarchy from a Small Extra Dimension", Phys. Rev. Lett. 83, 3370-3373 (1999), hep-ph/9905221.

[9] L. Randall and R. Sundrum, "An Alternative to Compactification”, Phys. Rev. Lett. 83, 4690-4693 (1999), hep-th/9906064.

[10] Globally, the topology can deviate from the simple product form. Not only can one have a "twisted" $K$-bundle over $M$, but even the local product structure can break down in places. At such points the dimensionally reduced spacetime will be singu- 
lar, betraying thereby the physical reality of the higher dimensions [11].

[11] R.D. Sorkin, “A Kaluza-Klein Monopole”, Phys. Rev. Lett. 51, 87-90 (1983); 54, 86 (1985).

[12] There is a potential confusion here that needs to be avoided, between the cosmological constant belonging to the effective 4dimensional description of the higher dimensional theory, and the true cosmological constant of the higher theory that would be reported by higher dimensional observers. The latter actually varies inversely to $\sqrt{N}$ when measured in higher dimensional Planck units, but it is not the quantity of phenomenological interest to us.

[13] The reasoning leading to this conclusion should transfer readily from the model of [7] to that of [9], with the 5-dimensional radius of curvature there playing the role of the compactification diameter. In other words $v$ here would correspond to $1 / k$ there. Notice in this connection that for the line-element, $d s_{5}^{2}=\exp (-2 k|y|) d s_{4}^{2}+d y^{2}$, employed in [9], the volume of the past of an event $x$ on the brane will approximately take the same form as we have used herein, as is not hard to verify: $\tilde{V} \approx V / k \approx V v$. (Here, $d s_{4}^{2}$ is the line-element of Minkowski space $M^{4}$.) I believe that similar comments will apply as well to the model of [8], but the translation between four and five dimensions seems less clear in that model. 